mode, a Hazard Score (HS) was calculated by multiplying the probability of occurrence (Remote $=1$, Uncommon $=2$, Occasional $=3$, Frequent $=4$ ) and severity of effect (Minor $=1$, Moderate $=2$, Major $=3$, Catastrophic $=4$ ). If $\mathrm{HS}>=8$, corrective actions were proposed. If $\mathrm{HS}<8$, failure mode was evaluated based on: lack of detection, criticality and absence of effective control measures. All data were collected in a validated worksheet.

Results A flow diagram was obtained. Twenty-seven failure modes were identified, and twenty had a HS $>=8$. Failure modes with the highest HS were: wrong dose calculation and wrong protocol (Prescribing); incorrect production protocol in the computer system and non-detection of wrong dose calculation (Pharmaceutical validation); wrong medicine is chosen, incorrect volume of drug added to diluent and labelling error (Compounding); Delivered to wrong nursing unit or patient (Dispensing). Corrective actions proposed were: policy of weighing patient for proper dose calculation, chemotherapy database updated, double checking, gravimetric control on prepared chemotherapy, procedures for proper patient identification (barcode identification system or radiofrequency dispensing system).

Conclusions FMEA contributes to the development of a very clear and shared vision of the chemotherapy process, taking into account different perspectives: oncologist, pharmacist, technician and nurse.

FMEA is a useful tool for identifying critical parts of the chemotherapy process, prioritising corrective actions, minimising potential risks and improving the quality and safety of patient care.

No conflict of interest.

\section{GRP-074 FREQUENCY OF VALPROIC ACID-INDUCED HYPERAMIMONEMIA IN ADULT PSYCHIATRIC SETTINGS}

doi:10.1136/ejhpharm-2013-000276.074

${ }^{1} \mathrm{~B}$ HUE, ${ }^{2} \mathrm{~N}$ Chaumartin, 'P Beauverie. 'EPS Paul Guiraud, Pharmacy, Villejuif, France; 2EPS Paul Guiraud, General Practitioner, Villejuit, France

Background Valproic acid (VPA) is widely prescribed by paediatric neurologists as an antiepileptic drug. VPA-induced hyperammonaemia can lead to encephalopathy and coma; it is well documented among the paediatric population. Severe urea cycle enzyme deficiencies are often revealed in early youth when VPA is administered. Such mild genetic deficiencies can remain unnoticed until adulthood and be discovered if VPA is taken for bipolar disorder.

Purpose To evaluate the frequency of VPA-induced hyperammonaemia in adult psychiatric settings and to sensitise the medical community to a potentially severe adverse effect of a widelyprescribed drug.

Materials and Methods The study was carried out a two-week period in a psychiatric hospital. It included every full-time hospitalised patient treated with VPA for at least 4 days (corresponding to 5 drug half-lives). Ammonia and VPA blood measurements were performed once and an electroencephalogram when ammonia exceeded $70 \mu \mathrm{M}$ (normal range: 10 to $35 \mu \mathrm{M}$ ). Ethics committee approval was obtained before starting the study.

Results 122 patients were included in this study. 68 patients $(55.8 \%)$ presented ammonia blood levels exceeding $35 \mu \mathrm{M}$ and 4 of them $(3.3 \%)$ exceeded $70 \mu \mathrm{M}$. One patient reached $118 \mu \mathrm{M}$ one week after VPA initiation. No encephalographic abnormalities were observed. No correlation was found between ammonia and total VPA levels. Different oral forms of VPA were used and this study showed that they affected VPA blood levels.

Conclusions VPA-induced hyperammonaemia is a frequent, generally well-tolerated, adverse effect. Ammonia blood level monitoring combined with clinical monitoring are essential to avoid hyperammaonemic encephalopathy. Communication within the hospital led to the medical community becoming aware of the problem and new monitoring recommendations were defined including initial ammonia level measurement after VPA initiation and biannual monitoring of this biological parameter. Total VPA level determination doesn't seem to be useful for predicting hyperammonaemia whereas the importance of measuring the free VPA has recently been highlighted.

No conflict of interest.

\section{GRP-075 GASTROPROTECTION WITH NON-STEROIDAL ANTI- INFLAMMATORY DRUGS AT HOSPITAL DISCHARGE: DO WE FOLLOW LOCAL GUIDELINES?}

doi:10.1136/ejhpharm-2013-000276.075

J Sotoca, M Rovira, C Codina, J Ribas. Hospital Clínic, Pharmacy Service, Barcelona, Spain

Background Studies have shown overuse of proton pump inhibitors (PPIs) that does not meet accepted criteria.

Purpose The aim of this study was to determine the prevalence and appropriateness of gastroprotection with PPIs in patients who were prescribed non-steroidal anti-inflammatory drugs (NSAIDs) at tertiary level hospital discharge.

Materials and Methods Data for this retrospective study were obtained from the pharmacy claims database 1-31 January 2012.

We identified patients under 65 years with a concomitant PPI and NSAID and who were not taking antiplatelet drugs, anticoagulants or steroids and revised the discharge report; we considered gastroprotection appropriate if it contained a history of ulcer disease, bleeding or gastroduodenal perforation or comorbidity or treatment indicated at the time of admission.

Results During January 2012 a total of 1776 patients were dispensed at least one prescription medicine at discharge.

388 patients were dispensed an NSAID and PPI, of whom 144 also received antiplatelet treatment, anticoagulants or steroids and for whom therefore gastroprotection was recommended. We analysed the age of the 244 remaining patients. 76 of them were $\geq 65$ years and then we also considered PPI gastroprotection appropriate. We reviewed the discharge report of the remaining 168 patients who were under 65 . The result of this analysis showed that 133 patients did not fit criteria for PPI use $34.3 \%$ of patients receiving NSAIDs and PPIs); gastroprotection was correct in 27 patients and the discharge report was not recovered in 8 patients $(2.1 \%)$.

Conclusions In this retrospective study, $63.6 \%$ of patients who were dispensed NSAIDs at discharge received appropriate PPI gastroprotection and $34.3 \%$ of patients received an unnecessary PPI prescription $(79.2 \%$ of patients under 65$)$.

Patient prescription at hospital discharge should be reviewed to prevent overuse of proton pump inhibitors, especially in patients under 65 years of age.

No conflict of interest.

\section{GRP-076 GASTROPROTECTIVE AGENTS IN THE EMERGENCY ROOM OF A TERTIARY-LEVEL HOSPITAL}

doi:10.1136/ejhpharm-2013-000276.076

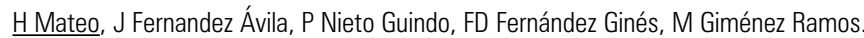
Torrecárdenas Hospital, Pharmacy, Almería, Spain

Background Gastroprotective agents are widely used in both hospital and community settings, and they are generally perceived as safe drugs.

Purpose To find out whether the prescription of anti-ulcer drugs in the Emergency Room (ER) accords with their approved indications, and the financial impact of their inappropriate use.

Materials and Methods Indications for use of proton pump inhibitors (PPIs) and H2 antagonists (via the Spanish Medicines 
Agency): gastro-duodenal ulcers (including NSAIDs and steroidrelated ulcers), reflux oesophagitis, Zollinger-Ellison's syndrome, and Helicobacter pylori eradication. Inclusion criteria: patients $>65$ years old on at least four home medicines and an anti-ulcer prescription in the ER. Pharmaceutical interventions were recorded and their degree of acceptance calculated. The cost resulting from drug misuse was calculated considering a mean stay in the unit of one day.

Results 111 patients, $70.2 \%$ male, median age 78.9 years-old [65-94]. 94.6\% of patients (92.9\% PPI, 1.7\% H2 antagonists) received one of these agents upon presentation $(95.5 \%$ of them were prescribed de novo), with intravenous pantoprazole the agent mainly involved ( $82 \%$ of cases). $29.7 \%$ of prescriptions did not meet the indications, while this percentage decreased to $12.5 \%$ upon ward admission. The pharmaceutical interventions were accepted in $16.2 \%$ of cases. Monthly, the estimated cost of the off-label use was $€ 1850$.

Conclusions Gastro-protection in the ER did not meet the criteria in nearly $1 / 3$ of patients. This contrasted with the poor acceptance of the pharmaceutical recommendations of discontinuation. The rationale might be the so-perceived harmless profile of these drugs with the short-term use. The rate of off-label prescriptions dropped to half upon ward admission, likely due to thorough revision by the prescriber. Since only patients at a higher risk of suffering from a medicines-related problem were included, the cost resulting from the misuse of anti-ulcer drugs was probably underestimated. In conclusion, forthcoming pharmacy policies should focus on improving the adherence to the indications of both widely-used and expensive drugs, given their financial and health-care impact.

No conflict of interest.

\section{GRP-077 GENERIC MYCOPHENOLATE MOFETIL IN HEART TRANSPLANT RECIPIENTS: IMPLEMENTATION OF ACTIVE PHARMACOVIGILANCE}

doi:10.1136/ejhpharm-2013-000276.077

'L Calixto, ${ }^{1 T}$ Laranjeira, 'A Mirco, ${ }^{2} \mathrm{MJ}$ Rebocho, ${ }^{1} \mathrm{~F}$ Falcão. ${ }^{1} \mathrm{CHLO}$ EPE - Hospital Santa Cruz, Hospital Pharmacy, Lisbon, Portugal; '² CHLO EPE - Hospital Santa Cruz, Transplant Heart Unit, Lisbon, Portugal

Background Immunossupressant drugs have an important role in the prophylaxis of transplant rejection, so they are considered 'critical dose drugs'. Use of a generic immunosuppressant represents a significant cost savings to the medical system. Since safety data for new medicines are always limited, post-marketing surveillance is essential to determine medicines' safety in real life use. With the introduction of generic mycophenolate mofetil (MMF) in CHLO, EPE-HSC, the pharmaceutical services (PHS) have implemented an MMF active pharmacovigilance programme (APP) for HT recipients.

Purpose To describe and quantify suspected adverse drug reactions (ADRs) identified with an APP implemented by the PHS.

Materials and Methods Between 11/2011 and 09/2012, all adult HT recipients who switched from innovator to the generic MMF were included in the MMF APP. This substitution was made under medical supervision and the pharmacist provided the patients with all necessary explanations. Subsequent pharmaceutical assessment was done with a questionnaire (in person or telephone), which identified demographic data, concomitant treatment and suspected ADRs.

Results 55 patients were included in the MMF APP, 78\% male, average age $55 \pm 13[22-76]$ years. 14 patients $(25 \%)$ reported ADRs at MMF switch. These patients had not experienced $\mathrm{ADR}$ s with the innovator drug. The most common $\mathrm{ADR}$ identified were diarrhoea (25\%), stomach ache (12.5\%) and asthenia (12.5\%). All ADRs notifications were reported to the Portuguese National Pharmacovigilance Unit.
Conclusions Most suspected ADRs identified corresponded to MMF's profile ADRs described in the summary of product characteristics. The switch to generic from innovator drug should have a surveillance strategy that includes medical monitoring, patient education and the contribution of all health professionals involved in the patient immunosuppressant regimen in order to create a system that allows adverse reactions to be detected, with the ultimate goal of maximising benefit and minimising risk by promoting safer use of medicines.

No conflict of interest.

\section{GRP-078 GUIDELINE FOR ALBUMIN USE: EFFECT ON COST SAVING}

doi:10.1136/ejhpharm-2013-000276.078

N Yurrebaso Eguilior, J González García, F Gutierrez Nicolas, S Duque Fernández de Vega, S González Ponsjoan, GJ Nazco Casariego. Hospital Universitario de Canarias, Pharmacy, La Laguna, Spain

Background Albumin has been widely used in clinical practise. While some of these indications are supported by the results of randomised studies, others are based only on clinical experience and have not been proved in prospective studies. Efforts should be made to define the indications for albumin use, so that patients gain the maximum benefit from its administration.

Purpose To evaluate the cost saving obtained by the implementation of a guideline for albumin use in a 737-bed hospital.

Materials and Methods Retrospective study that compared albumin use in two periods: July-September 2012 vs. July-September 2011. In June 2012 the guideline for albumin use was distributed to the medical staff. Physicians were requested to complete a form for each albumin order indicating the type and amount of albumin, the clinical service, and the indication for use. Albumin use data and costs were obtained from pharmacy service management system $\left(\mathrm{SAP}^{\circledR}\right)$ and were tabulated using the Exce ${ }^{\circledR}$ software.

Results The total amount of albumin ordered during the study period was $29.360 \mathrm{~g}(€ 63,246)$ vs. $53.195 \mathrm{~g}(€ 108,617)$ for the same period during 2011, which means a reduction of $45 \%$. In terms of cost, the saving obtained amounted to $€ 45,371$ (58\%). The albumin use by specialty had also changed; a major decrease in use of albumin was observed for Anaesthesiology 4,000 g (75\%), General Surgery 3,080 g (65\%), Nephrology 4,900 g (64\%), Internal Medicine $3,860 \mathrm{~g}(56 \%)$, Haematology 1,410 g (53\%) and Digestive 1,400 g $(30 \%)$. On the other hand, Haemodialysis significantly increased its use of albumin to $2,805 \mathrm{~g}$ (65\%), although within the approved indication of plasmapheresis.

Conclusions An albumin use guideline with restrictions focused on albumin prescriptions had suficient efficacy to reduce consumption and save cost. In our hospital guideline the cost of implementation decreased a 58\% (€181,484 per year).

No conflict of interest.

\section{GRP-079 GUIDELINES FOR CHEMOTHERAPY EXTRAVASATION}

doi:10.1136/ejhpharm-2013-000276.079

${ }^{1} \mathrm{M}$ Morgado, ${ }^{2} \mathrm{M}$ Mendes, ${ }^{1} \mathrm{R}$ Oliveira, ${ }^{\prime} \mathrm{S}$ Morgado. 'Hospital Centre of Cova da Beira, Pharmaceutical Services, Covilhã, Portugal; ${ }^{2}$ University of Beira Interior, Health Sciences Faculty, Covilhã, Portugal

Background The administration of intravenous cytotoxic drugs plays a key role in cancer treatment and due to the overall increase in intravenous chemotherapy there has been an increasing incidence of chemotherapy extravasation. Therefore, it is advisable to have updated guidelines that direct the treatment of intravenous cytotoxics extravasation.

Purpose To develop guidelines for the treatment of cytotoxic extravasation, which contained the management algorithms, 